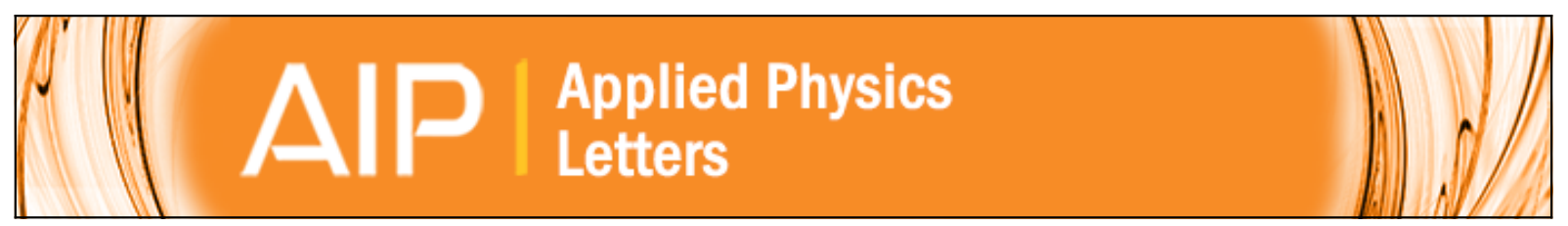

\title{
Coupling polariton quantum boxes in sub-wavelength grating microcavities
}

Bo Zhang, Sebastian Brodbeck, Zhaorong Wang, Martin Kamp, Christian Schneider, Sven Höfling, and Hui Deng

Citation: Applied Physics Letters 106, 051104 (2015); doi: 10.1063/1.4907606

View online: http://dx.doi.org/10.1063/1.4907606

View Table of Contents: http://scitation.aip.org/content/aip/journal/apl/106/5?ver=pdfcov

Published by the AIP Publishing

\section{Articles you may be interested in}

All-dielectric GaN microcavity: Strong coupling and lasing at room temperature

Appl. Phys. Lett. 102, 101113 (2013); 10.1063/1.4795019

Single-wavelength, all-optical switching based on exciton-polaritons

Appl. Phys. Lett. 101, 131104 (2012); 10.1063/1.4754575

Oriented polaritons in strongly-coupled asymmetric double quantum well microcavities

Appl. Phys. Lett. 98, 081111 (2011); 10.1063/1.3559909

Slow light at terahertz frequencies in surface plasmon polariton assisted grating waveguide

J. Appl. Phys. 106, 103715 (2009); 10.1063/1.3260236

Polariton quantum boxes in semiconductor microcavities

Appl. Phys. Lett. 88, 061105 (2006); 10.1063/1.2172409

\section{Model PS-100}

Tabletop Cryogenic Probe Station
Lake Shore CRYOTRONICS
An affordable solution for a wide range of research 


\title{
Coupling polariton quantum boxes in sub-wavelength grating microcavities
}

\author{
Bo Zhang, ${ }^{1}$ Sebastian Brodbeck, ${ }^{2}$ Zhaorong Wang, ${ }^{1}$ Martin Kamp, ${ }^{2}$ Christian Schneider, ${ }^{2}$ \\ Sven Höfling, ${ }^{2,3}$ and Hui Deng ${ }^{1, a)}$ \\ ${ }^{1}$ Department of Physics, University of Michigan, Ann Arbor, Michigan 48109, USA \\ ${ }^{2}$ Technische Physik, Physikalisches Institut and Wilhelm Conrad Röntgen-Research Center for Complex \\ Material Systems, Universität Würzburg, Am Hubland, D-97074 Würzburg, Germany \\ ${ }^{3}$ SUPA, School of Physics and Astronomy, University of St Andrews, St Andrews KY16 9SS, United Kingdom
}

(Received 8 December 2014; accepted 21 January 2015; published online 4 February 2015)

\begin{abstract}
We report the construction of decoupled, coupled, and quasi-one dimensional polariton systems from zero dimensional polariton quantum boxes using microcavities with sub-wavelength gratings as the top mirror. By designing the tethering patterns around the suspended sub-wavelength gratings, we control the coupling between individual quantum boxes through different optical potentials. Energy levels and real-space or momentum space distributions of the confined modes were measured, which agreed well with simulations. (C) 2015 AIP Publishing LLC.
\end{abstract}

[http://dx.doi.org/10.1063/1.4907606]

Exciton-polariton condensation in two dimensional (2D) systems has been widely observed and researched. ${ }^{1-6}$ The polariton system may also provide an accessible venue for research on lattice physics ${ }^{7}$ and quantum optical circuits, ${ }^{8-10}$ which requires the capability to confine it into lower dimensions and couple multiple ones. Along this line, 2D polaritons have been loaded into lattice potentials using metal patterns on the top surface of the cavity. ${ }^{11-14}$ Yet the patterns are typically ten wavelengths away from the center of the cavity, hence the potential barrier height is limited and lower dimensional polaritons are hard to achieve. Alternatively, stronger lateral confinement has been reported in pillar structures created from a 2D cavity by deep plasma etching, producing polaritons in lower dimensions and lattice potentials. ${ }^{15-18}$ Yet the method requires plasma bombardment over a long time and creates a large side surface surrounding the active media and the cavity. Hence, it is challenging to control the loss and decoherence of the excitons and polaritons. In this letter, we demonstrate a flexible and non-invasive method to create polariton quantum boxes and control coupling among multiple quantum boxes, by the use and design of a sub-wavelength grating (SWG) as the top cavity mirror.

Integration of SWGs into a polariton-cavity has unique advantages. The SWGs are air-suspending gratings with periods smaller than the optical wavelength. ${ }^{19}$ Similar to the most widely used distributed Bragg reflectors (DBRs), dielectric SWGs are grown epitaxially with the rest of the device, resulting in a monolithic cavity with high crystal quality. Unlike DBRs, SWGs can achieve high reflectance while only one tenth in thickness, ${ }^{20,21}$ bringing the designable SWG mirror very close to the center of the cavity. Together with the design flexibility of the grating parameters, when operating in the strong-coupling regime, SWGs may enable unprecedented control of the polariton modes, without modifying the rest of the cavity or the active media layer. Polariton lasing has been demonstrated in a zerodimensional (OD) SWG-DBR hybrid cavity with polarization

\footnotetext{
${ }^{a)}$ Electronic mail: dengh@umich.edu
}

selectivity. ${ }^{22,23}$ Dispersion engineering of the photon and polariton modes has been proposed. ${ }^{24}$ Here, we present the study of multiple, coupled SWG cavities. We show that, by design of the tethering patterns around the SWGs, coupling among multiple 0D-polariton quantum boxes can be readily achieved, leading to de-coupled, coupled, and quasi-1D polariton systems. This enables advanced mode engineering and provides the building blocks for polariton-based quantum simulators and quantum circuits. ${ }^{8-10}$

Using the SWG, strong lateral confinement of polariton to $0 \mathrm{D}$ is achieved by reducing the lateral size of the SWG to a few microns. This is because the sudden change in the reflectance from the SWG region to the planar region surrounding it results in a large effective potential at the lateral boundaries of the SWG. The SWGs are suspended above an air gap and tethered to the surrounding planar part of the wafer. To create additional potentials and to control the coupling among OD SWG-cavities, we place through-etched long rectangular slots in the tether, which changes the boundary condition of the cavity and creates effectively potential barriers for the cavity modes. The potential is centered at the center of the slots, with its width and height controlled by the length of the slot. By arranging the positions and changing the lengths of the slots, we can create different effective potentials for the photon modes. In the strongcoupling regime, the photon potential is directly transcribed to the polaritons via the eigen energy equation

$$
E_{\mathrm{UP}, \mathrm{LP}}=\frac{E_{\mathrm{ph}}+E_{\mathrm{exc}}}{2} \pm \frac{\sqrt{\left(E_{\mathrm{ph}}-E_{\mathrm{exc}}\right)^{2}+(\hbar \Omega)^{2}}}{2},
$$

where $E_{\mathrm{UP}, \mathrm{LP}}$ are the eigen energies of upper and lower polaritons, respectively, $E_{\mathrm{ph}}$ is the cavity photon energy, $E_{\mathrm{exc}}$ is the exciton energy, and $\hbar \Omega$ is the coupling strength. Using this method, we demonstrate three polariton devices in different potentials. We first demonstrate the creation of two $0 \mathrm{D}$ polariton quantum boxes from a long quasi-1D wire, by placing a long slot in the tether to create a wide Gaussian potential barrier in the middle of the wire. Then, using the same SWG, but changing the slot patterns in the tether to 
reduce the barrier width and height, we create a coupled polariton systems in a triple-well potential. Finally, by increasing the number of the $0 \mathrm{D}$ polariton quantum boxes while maintaining the coupling, we create a lattice potential for the polaritons. Polariton energy bands were observed.

The devices we used were grown by molecular beam epitaxy. The planar epitaxial sample consists of an $\mathrm{Al}_{0.15} \mathrm{Ga}_{0.85} \mathrm{As}$ layer on top, followed by an $\mathrm{Al}_{0.85} \mathrm{Ga}_{0.15} \mathrm{As}$ sacrificial layer, two and half pairs of $\mathrm{AlAs} / \mathrm{AlGaAs}$ top DBR to protect the cavity region, a $\lambda / 2$ cavity with 12 quantum wells embedded, and 30 pairs of DBR at the bottom. The SWGs were created by electron beam (ebeam) lithography and plasma etching of the top layer, followed by a selective chemical etching to remove the sacrificial layer. The gratings all have a period of $520 \mathrm{~nm}$ and a duty cycle of $40 \%$. The exciton energy was $E_{\text {exc }}=1.551 \mathrm{eV}$ and the lower and upper polariton splitting was $\hbar \Omega=11 \mathrm{meV}$ in the SWGDBR cavities. More details of the fabrication and sample properties can be found in Zhang et al. ${ }^{22}$

To characterize the confinement and coupling of the devices, real space and Fourier space distributions of the polariton modes were measured via photoluminescence (PL) while the sample was kept at the temperature of $10 \mathrm{~K}$. A pulsed pumping laser was used to excite the sample nonresonantly from the normal direction at the wavelength of $740 \mathrm{~nm}$, pulse width of $\sim 150 \mathrm{fs}$, and repetition rate of $80 \mathrm{MHz}$. For real space imaging, the pumping laser was focused to a spot of $\sim 50 \mu \mathrm{m}$ in diameter, so as to excite the entire device. For Fourier space imaging, the pumping laser spot was centered on the device with a diameter of $\sim 3 \mu \mathrm{m}$. The PL signals were collected by an objective lens with a numerical aperture of 0.55 . All the SWGs were optimized for the TE polarization; hence a polarizer filtered the TE signals after the objective lens. A group of lens relay was used to direct the signal into a $0.5 \mathrm{~m}$ spectrometer with a liquid nitrogen cooled coupled charge device detector, where the spectrally resolved real-space and Fourier space images of the PL were recorded.

The first two devices had the same SWG-wire of $7.5 \mu \mathrm{m}$ in width and $30 \mu \mathrm{m}$ in length, while different patterns in the tethers created different potentials for the polaritons. In the first device, we created two separate OD polariton quantum boxes by placing a pair of long slot of $8.5 \mu \mathrm{m}$ in length, in the middle of both of the top and bottom tethers, as shown in the scanning electron microscope (SEM) image in Fig. 1(a). The resulting lower polariton (LP) modes were measured via spectrally resolved real space imaging, as shown in Fig. 1(b). Two spatially separated groups of LP modes were observed, with identical discrete energy levels. It suggests that the slots create a potential barrier, confining the LPs to two OD quantum boxes to its two sides.

We model the potential barriers created by the slots by Gaussian functions: $A \times \exp \left(-\frac{x-x_{0}}{B}\right)^{2}$. Here, $x$ is the position along the SWGs' longer dimension, measured from the center of the SWG. $x_{0}$ is given by the center of the slot. $A$ and $B$ correspond to the height and width of the barrier, respectively, and are positively correlated with the width of the slot. For the long slot placed at the center of the wire, $x_{0}=0$, $A=10 \mathrm{meV}$, and $B=4 \mu \mathrm{m}$. We model the effective harmonic potential at the two ends of the SWG-wire as (a)

(c)

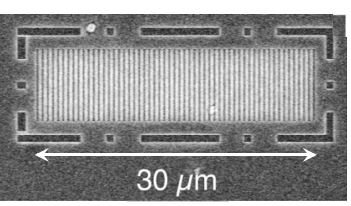

(b)

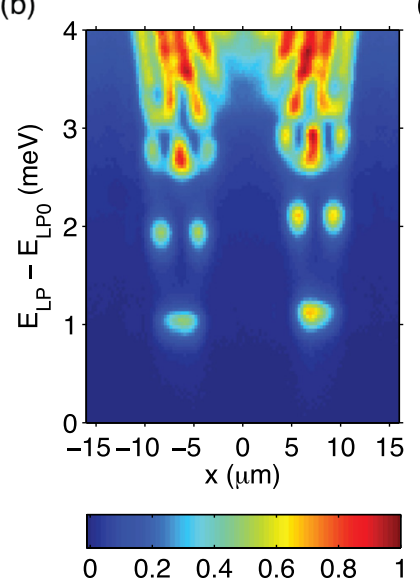

(d)

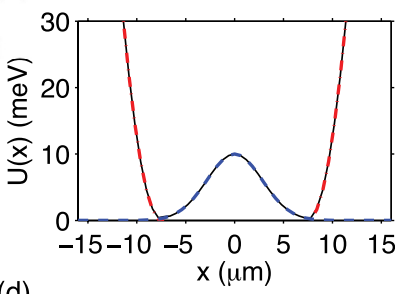

)

FIG. 1. Two decoupled OD polariton systems. (a) A top-view SEM image of the device. (b) The spectrally resolved real space image of the LP PL, showing two spatially separate groups of discrete modes. $\mathrm{E}_{\mathrm{LPO}}$ is the LP energy without confinement. (c) Effective photon potentials in the device. The total potential (the black solid line) is the sum of the Gaussian barrier (the blue dashed line) and the harmonic potential (the red dotted-dashed line) towards edges of the device. (d) The calculated real-space distribution of the the LP wave functions using the total potential in (c).

$U(x)=a(x-d)^{2}$. Here, $a$ is $2 \mathrm{meV}$ and $d$ is $\pm 7.2 \mu \mathrm{m}$. The total potential along the wire is the sum of all the Gaussian barriers and the two harmonic potentials. The profile of each contributing potential and the total potential is plotted in Fig. 1(c). Using the total potential, we can first calculate the confined photon energy levels and the corresponding real-space wave functions. Then the LP energy levels can be calculated using Eq. (1). The resulting polariton energy levels and the corresponding spatial wave functions are shown in Fig. 1(d), which match very well the experimental results in Fig. 1(b).

In the second SWG device, we demonstrate coupling among three OD polariton quantum boxes. We implement this using two closely spaced and shallower potential barriers, created by two shorter slots $7 \mu \mathrm{m}$ apart, as shown in the SEM image in Fig. 2(a). The resulting LP modes were shown in Fig. 2(b). Similar to the first device, the energy levels are discrete, showing the OD nature of each constituent quantum boxes. At the same time, unlike having separate groups of LPs in the first device, LPs show renormalized energy levels and distinct features resulting from coupling among the three quantum boxes, or, tunneling through the barriers. For example, there exists a common ground state at a lower energy than the ground states in the first device. The spatial wavefunction of the ground state has an anti-node at the center of the device but no nodes. The first and second excited states are very closely spaced in energy, different from either three uncoupled quantum boxes or an unmodulated quantum wire.

To model the potentials, we use the same method as for the first device. Gaussian functions are used for the barriers created by the slots and harmonic potentials are used towards 
(a)

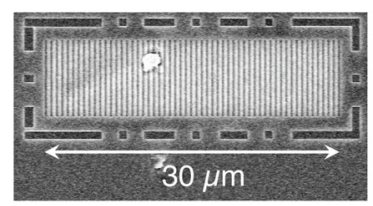

(b)

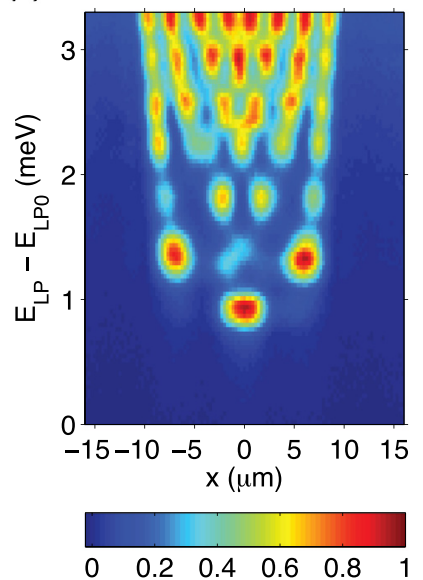

(c)

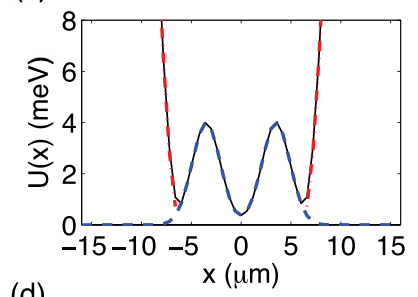

(d)

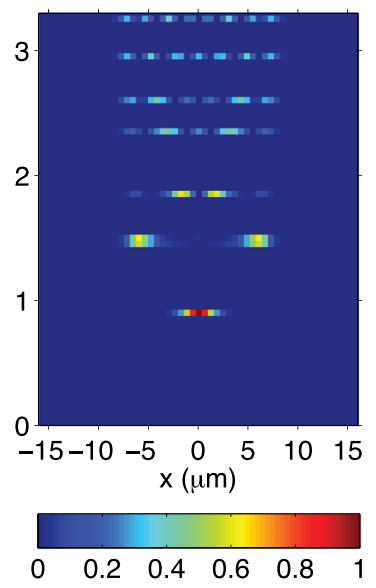

FIG. 2. A coupled polariton systems from three 0D polariton quantum boxes. (a) A top-view SEM image of the device. (b) The spectrally resolved real space image of the LP. (c) Effective photon potentials in the device, including the total potential (the black solid line), two shallow Gaussian barriers (the blue dashed lines) and two harmonic potentials (the red dotteddashed line). (d) The calculated real-space distribution of the the LP wave functions using the total potential in (c). It shows a non-degenerate ground state, nearly degenerate first and second excited states, and a few higher excited states. The results match well with (b).

the two ends of the wire, as shown in Fig. 2(c). The two main barriers are centered at $x_{0}= \pm 3.5 \mu \mathrm{m}$, with a smaller height and width of $A=4 \mathrm{meV}$ and $B=2 \mu \mathrm{m}$ corresponding to the shorter lengths of the slots. The calculated energy levels and real-space distributions of the wave functions are shown in Fig. 2(d). Due to the shallower barrier and closer spacing of the potential wells, quantum tunneling between the potential wells is pronounced. The ground state and the first several excited states of the coupled system match very well with the measured results in Fig. 2(b).

Finally, we implement a quasi-1D polariton lattice by extending the two coupled OD polariton quantum boxes in the second device to nine coupled ones. We use a long SWG-wire of $60 \mu \mathrm{m}$ in length and create the periodic potential with a periodic slot-pattern in the tether. The slots are $3 \mu \mathrm{m}$ wide and $\sim 7 \mu \mathrm{m}$ apart, same as in the second device. The SEM image of the device is shown in Fig. 3(a). The measured energy-wavenumber dispersions of the LP modes are shown in Fig. 3(b). Energy gaps can be identified at the edges of the first Brillouin zone at $\pm \pi / 7 \mu \mathrm{m}^{-1}$ and $\pm 2 \pi /$ $7 \mu \mathrm{m}^{-1}$, corresponding to the lattice constant of $7 \mu \mathrm{m}$. Up to three LP dispersion curves were observed in the repeated zone scheme. At higher energies, additional branches were also observed due the one-dimensional nature of the wire. The results agree well with the calculated dispersions as shown in Fig. 3(c).

To conclude, we have demonstrated the construction of confinement potentials for polaritons by design of the tether

(a)

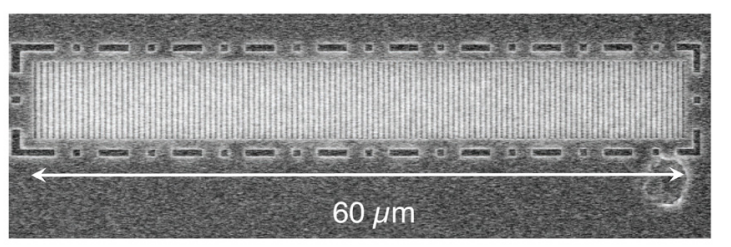

(b)

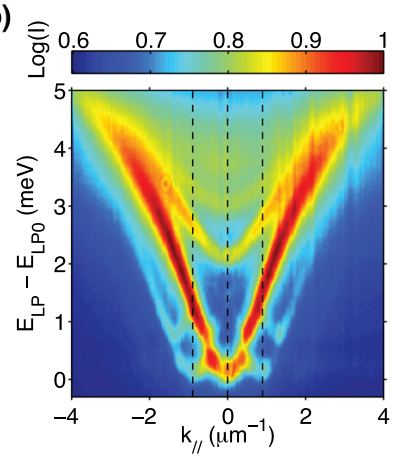

(c)

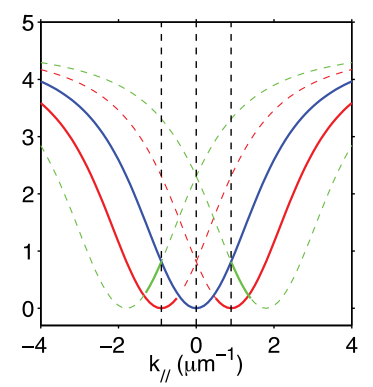

FIG. 3. A 1D polariton lattice system. (a) A top-view SEM image of the device. (b) The measured energy-wavenumber dispersion of the LP. (c) The calculated energy-wavenumber dispersion of the LPs in a lattice potential with a lattice period of $7 \mu \mathrm{m}$.

patterns in SWG-DBR cavities. De-coupled, coupled, and lattice polariton systems have been created. Together with the flexibility to design the SWGs themselves, such as polarization selectivity, ${ }^{20,22}$ resonance tuning, ${ }^{21}$ and dispersion engineering, ${ }^{24}$ the SWG-DBR cavities provide unique opportunities for matter-wave circuits ${ }^{8,9}$ and many body physics in open systems. ${ }^{10}$

B.Z., Z.W., and H.D. acknowledge support from the National Science Foundation under Award No. DMR 1150593 and Air Force Office of Scientific Research under Award No. FA9550-12-1-0256. S.B., M.K., C.S., and S.H. acknowledge support by the State of Bavaria, Germany. S.H. thanks furthermore the Royal Society and the Wolfson Foundation for their support. SWG device fabrication was completed at the Lurie Nanofabrication Facility, which is part of the National Science Foundation NNIN network.

${ }^{1}$ H. Deng, G. Weihs, C. Santori, J. Bloch, and Y. Yamamoto, Science 298, 199 (2002).

${ }^{2}$ J. Kasprzak, M. Richard, S. Kundermann, A. Baas, P. Jeambrun, J. M. J. Keeling, F. M. Marchetti, M. H. Szymańska, R. André, J. L. Staehli, V. Savona, P. B. Littlewood, B. Deveaud, and L. S. Dang, Nature 443, 409 (2006).

${ }^{3}$ R. Balili, V. Hartwell, D. Snoke, L. Pfeiffer, and K. West, Science 316, 1007 (2007).

${ }^{4}$ S. Christopoulos, G. Von Högersthal, A. Grundy, P. Lagoudakis, A. Kavokin, J. Baumberg, G. Christmann, R. Butté, E. Feltin, J.-F. Carlin, and N. Grandjean, Phys. Rev. Lett. 98, 126405 (2007).

${ }^{5}$ S. Cohen-Kena and S. Forrest, Nat. Photonics 4, 371 (2010).

${ }^{6}$ H. Deng, H. Haug, and Y. Yamamoto, Rev. Mod. Phys. 82, 1489 (2010).

${ }^{7}$ M. J. Hartmann, F. G. S. L. Brandão, and M. B. Plenio, Nat. Phys. 2, 849 (2006).

${ }^{8}$ T. Liew, A. Kavokin, and I. Shelykh, Phys. Rev. Lett. 101, 016402 (2008). ${ }^{9}$ T. C. H. Liew, A. V. Kavokin, T. Ostatnický, M. Kaliteevski, I. A. Shelykh, and R. A. Abram, Phys. Rev. B 82, 033302 (2010).

${ }^{10}$ I. Carusotto and C. Ciuti, Rev. Mod. Phys. 85, 299 (2013).

${ }^{11}$ C. W. Lai, N. Y. Kim, S. Utsunomiya, G. Roumpos, H. Deng, M. D. Fraser, T. Byrnes, P. Recher, N. Kumada, T. Fujisawa, and Y. Yamamoto, Nature 450, 529 (2007). 
${ }^{12}$ N. Y. Kim, K. Kusudo, C. Wu, N. Masumoto, A. Löffler, S. Höfling, N. Kumada, L. Worschech, A. Forchel, and Y. Yamamoto, Nat. Phys. 7, 681 (2011).

${ }^{13}$ N. Y. Kim, K. Kusudo, A. Löffler, S. Höfling, A. Forchel, and Y. Yamamoto, New J. Phys. 15, 035032 (2013).

${ }^{14}$ N. Y. Kim, K. Kusudo, A. Löffler, S. Höfling, A. Forchel, and Y. Yamamoto, Phys. Rev. B 89, 085306 (2014).

${ }^{15}$ T. Gutbrod, M. Bayer, A. Forchel, and J. P. Reithmaier, Phys. Rev. B 57, 9950 (1998).

${ }^{16}$ D. Bajoni, P. Senellart, E. Wertz, I. Sagnes, A. Miard, A. Lemaître, and J. Bloch, Phys. Rev. Lett. 100, 1 (2008).

${ }^{17}$ E. Wertz, L. Ferrier, D. D. Solnyshkov, R. Johne, D. Sanvitto, A. Lemaître, I. Sagnes, R. Grousson, A. V. Kavokin, P. Senellart, G. Malpuech, and J. Bloch, Nat. Phys. 6, 860 (2010).
${ }^{18}$ M. Galbiati, L. Ferrier, D. Solnyshkov, D. Tanese, E. Wertz, A. Amo, M. Abbarchi, P. Senellart, I. Sagnes, A. Lemaître, E. Galopin, G. Malpuech, and J. Bloch, Phys. Rev. Lett. 108, 1 (2012).

${ }^{19}$ C. F. R. Mateus, S. Member, M. C. Y. Huang, Y. Deng, A. R. Neureuther, and C. J. Chang-Hasnain, IEEE Photonics Technol. Lett. 16, 518 (2004).

${ }^{20}$ M. C. Huang, Y. Zhou, and C. J. Chang-Hasnain, Nat. Photonics 1, 119 (2007).

${ }^{21}$ M. C. Y. Huang, Y. Zhou, and C. J. Chang-Hasnain, Nat. Photonics 2, 180 (2008).

${ }^{22}$ B. Zhang, Z. Wang, S. Brodbeck, C. Schneider, M. Kamp, S. Höfling, and H. Deng, Light: Sci. Appl. 3, e135 (2014).

${ }^{23}$ J. Fischer, S. Brodbeck, B. Zhang, Z. Wang, L. Worschech, H. Deng, M. Kamp, C. Schneider, and S. Höfling, Appl. Phys. Lett. 104, 091117 (2014).

${ }^{24}$ Z. Wang, B. Zhang, and H. Deng, "Dispersion Engineering of Vertical Microcavities using Sub-wavelength Gratings,” Phy. Rev. Lett. (in press). 